\title{
CORRECTIONS
}

\section{Brackets (parentheses) in formulas}

The previous version of this Statistics Notes article by Douglas G Altman and J Martin Bland in the Research Methods and Reporting section (BMJ 2011;343:d570, doi:10.1136/bmj.d570) was replaced with a revised version because of problems with formatting the online version. The revised version is correctly formatted, with the example formulas now integrated into the body of the text, and the references have working hyperlinks. In addition, a reader alerted us and the authors to an erroneous equation in example 1 of the first version of article (see www. bmj.com/content/343/bmj.d570.full/reply\#bmj_el_268526 and Huber-Wagner et al. Lancet 2009;373:1455-61). Although the citation of the original source's equation was correct, there was an error in the subsequent text. This example has now been omitted from the revised article.

Cite this as: $B M J$ 2011;343:d5296 